\title{
Image Information Mining - Exploration of Earth Observation archives
}

\author{
Mihai Datcu, Wessling, Klaus Seidel, Zürich
}

\section{Introduction}

Information mining opens new perspectives and a huge potential for information extraction from large volumes of heterogeneous images and the correlation of this information with the goals of applications.

The image archives are heterogeneous, huge data repositories, they are high complexity sources of valuable information, e.g. the Earth Observation data archives contain millions of optical, radar and other types of images and data. The exploration of their content is not an easy task. Among the promising methods proposed in the recent years are those for data and information mining. However, accessing the image information content involves highly complex problems arising primarily from the huge volume of data, the extensive information content, and the subjectivity of the user interpretation. The present article analyzes those Image Information Mining methods seen as information transmission problems: the source of information is an image archive, the receiver is the community of users. Data and information mining are exploratory processes focusing on the techniques for analyzing and combining raw data and detecting patterns and regularities within the data set. The success of the exploratory information search depends on the capacityof the search engine to capture and describe the full complexity of the data. Thus we make use of concept integrating multiple methods: information theory, stochastic modelling, Bayesian inference, machine learning. Information theory deals with encoding data in order to transmit it correctly and efficiently. The theory of stochastic processes and machine learning deals with estimating models of data and predicting future observations. There is a relationship between these fields: as the encoding of the data by the probabilistic model is the most compact and the most descriptive, there is a fundamental link between information and probabilistic models. This link is the base for the implementation of optimal algorithms for information extraction, detecting causalities, and for the design of information systems implementing image information mining functions. The article presents and analyzes several methods for mining the information content of large image repositories and demonstrates image mining functions, like search by example, search by data model, exploration in the scale space and image complexity, knowledge acquisition, and adapting to the user conjecture.

\section{From content based image retrieval to mining image information}

The continuous expansion of multimedia into all sectors of activity faces us with a double explosion:

- the number of image data sets,

- the data size and information variability of each image,

e.g. with a digital camera we can produce $10 \mathrm{~GB}$ of images during a three weeks holiday, a satellite sensor can produce $100 \mathrm{~GB}$ per day.

It has been known for many years that classical image file text annotation is prohibitive for large data bases. The last decade is marked by important research efforts in the development of Content Based Image Retrieval (CBIR) concepts and systems (VeLTKAmp et al. 2001). Images in an archive are searched by their visual similarity with respect to color, texture or shape characteristics. With the continuous development of image size and information content, CBIR was soon no longer satisfactory and Region Based Information Retrieval (RBIR) systems were developed (VeLtKAMP et al. 2001). Each image is segmented and individual objects are indexed by primitive attributes like color, texture and shape. Thus, RBIR is a solution to deal with the variability of image content.

However, both CBIR and RBIR are computer centered approaches, i.e. the concepts could only marginally or not at all adapt to the user needs. Consequently, the image retrieval systems were equipped with relevance feedback functions (Cox et al. 1996). The systems are designed to search images similar to the user conjecture. The algorithms are based on analyses of the probabilities of an image relative to the search target. A feedback mechanism taking this part into account is introduced.

Another interesting approach was developed based on a learning algorithm to select and combine feature grouping and to allow users to give positive and negative examples. The method refines the user interaction and enhances the quality of the queries (MinKa \& PICARD 1997).

Both previously mentioned concepts are first steps to include the user in the search loop. Thus, they are information mining concepts developed during the trend of designing human centered systems (Insert $1)$. 
Where is the information? - The information is in models. Information about an unknown variable $\mathrm{x}$ can be obtained if we are able to model the incertitude of the variable. If the probalilistic model is $\mathrm{p}(\mathrm{x})$, the information ii, then

$$
\mathrm{i}(\mathrm{x})=\log \left(\frac{1}{\mathrm{p}(\mathrm{x})}\right)
$$

In the case of mining information in large Earth Observation (EO) archives, we need to build hypotheses about the possible models describing the data. We base our model on the prior knowledge of the image formation process, thus being able to describe the causalities which link the physical parameters of the scene to the image content and further to the user understanding in terms of symbols and semantics he or she is using.

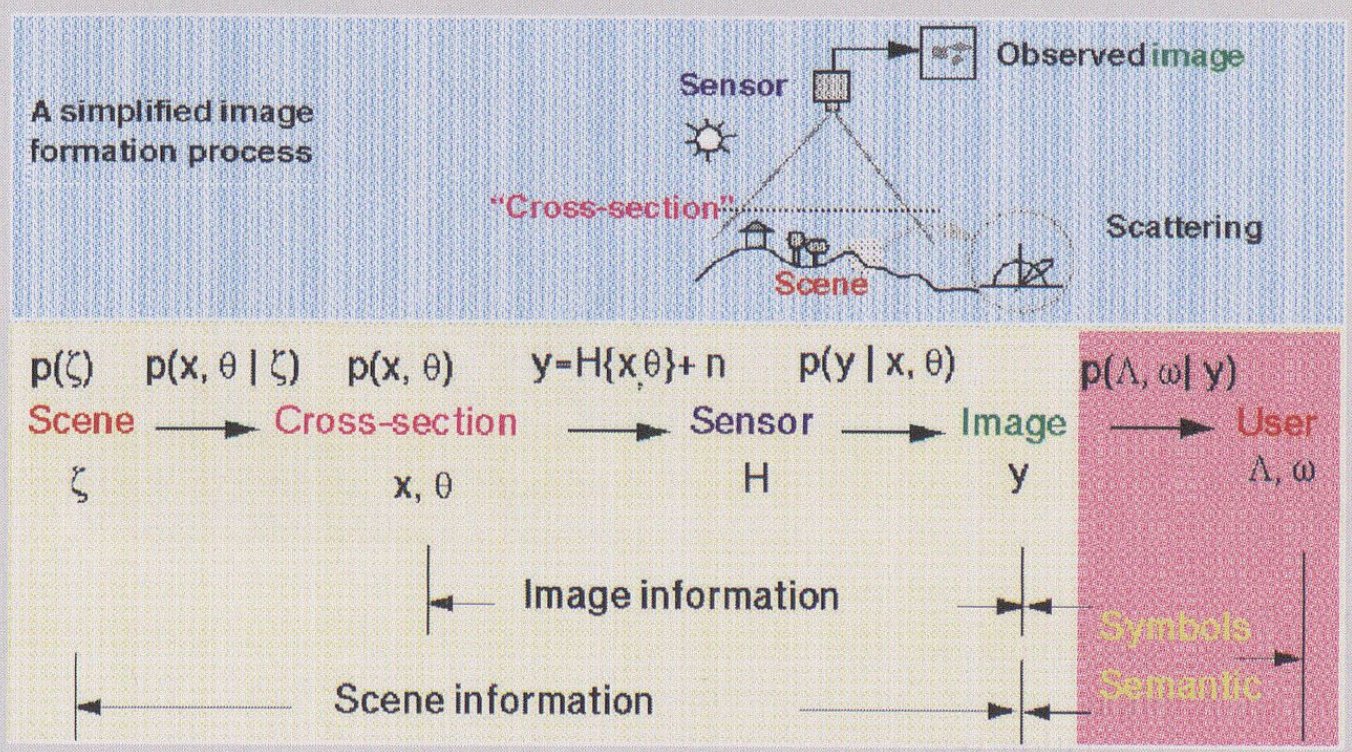

Insert 1: The generic image formation process upgraded with the user models Traditionally, in the interpretation of $E O$ data two operations are performed:

1. image understanding which has as goals the classification and/or recognition of 2-dimensional structures in the data,

2. scene understanding with the extraction of scene parameters from the 2-dimensional image observation.

In the case of image information mining the problem faced is more complex. The large amount of data implies a broad diversity of image structures and therefore a much larger class of models is needed.

In our approach we propose a quasi-complete image content description for optical data with the use of

1. spectral signatures

2. texture

3. multiscale structural analyses

4. geometrical features.

However, other sensor data require different types of models, e.g. synthetic aperture radar data needs specific modelling of the coherent phenomena.

Bild-Gewinnungsprozess mit Berücksichtigung der Nutzer Le processus de formation de l'image générique adapté aux modèles de l'utilisateur Graphics: Image Information Mining (Cooperation DLR/ETHZ) 


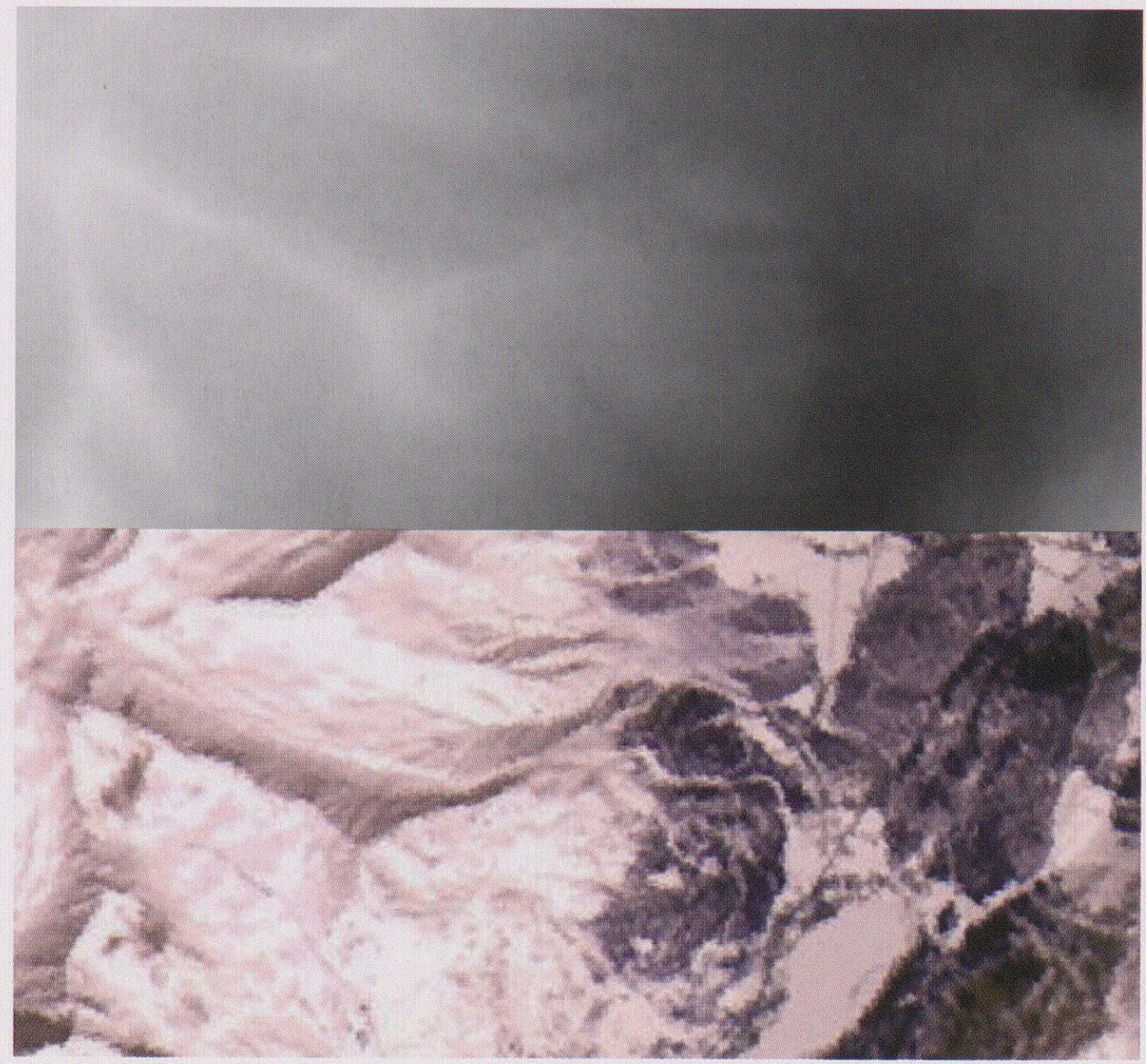

Fig. 1: Quantitative versus pictorial information

Explanation: Top: Visualization of a Digital Elevation Model (DEM) data set of Davos, Switzerland. The information on terrain height is contained in the pixel intensity, the information is quantitative and is not rich in visual meaning. Bottom: Satellite image (Landsat TM) of the same area. The information is pictorial, thus aggregation of colors, textures and geometrical objects at different scales makes it possible to understand the scenery of an alpine ski resort.

Quantitative oder bildmässige Information

Le quantitatif contre le contenu imagé de l'information

Source: Landsat-TM

\section{Images and image information}

Compared with Data Mining the field of Image Information Mining reaches much higher complexity resulting from:

- the huge volume of data ( $\mathrm{Tb}$ to $\mathrm{Pb}$ ),

- the variability and heterogeneity of the image data (diversity of sensors, time or conditions of acquisition, etc.),

- the image content, its meaning often being subjective, depending on the users interest,

- the large range of user interests, semantics and contextual (semiotic) understanding.

In general, when we talk of images we understand pic- tures, thus relating it to the (human) visual perception and understanding. A picture is characterized by its primitive features such as color, texture and shape at different scales. Its perception and understanding is in the form of symbols and semantics in a certain semiotic context (ZHANG et al. 2001).

However, the concept of image is beyond the pictorial understanding. Images are multidimensional signals, like computer tomography, hyperspectral images or results of simulations. They are communicated to users via 2 -dimensional visual projections. These images can contain quantitative, objective information, as acquired by an instrument.

In Fig. 1, an example is presented for the visualization 


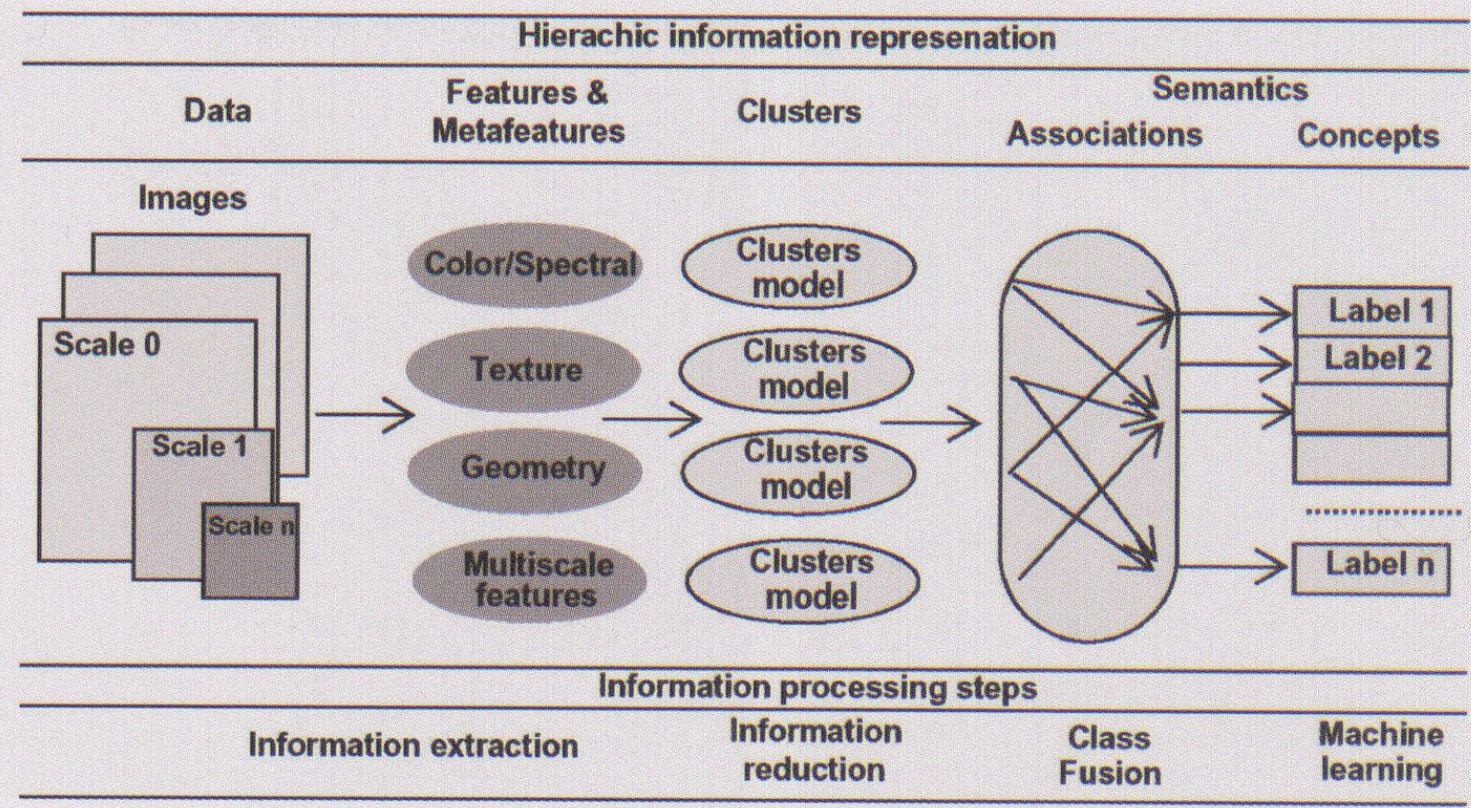

Fig. 2: The hierarchical representation of the image information content, and the causalities to correlate the user conjecture to the image content

Explanation: The key elements are the quasi-complete image signal description by extraction of the elementary features, the data reduction by clustering, thus inducing also a measure of some similarity over the feature space, the utilization of the cluster models as elements of an abstract vocabulary which in an interactive process enables the learning of the semantics of the target and the user conjecture.

Hierarchische Darstellung des Informations-Inhalts eines Bildes und die Zusammenhänge zwischen den NutzerAnnahmen und dem Bildinhalt.

La représentation hiérarchique du contenu de l'information d'images, et les causalités destinées à corréler la conjecture d'utilisateur avec le contenu de l'image.

Graphics: Image Information Mining (Cooperation DLR/ETHZ)

of a data set of a Digital Terrain Model (DEM) in comparison with a color rendered satellite image of the same Alpine region. The visual information in the DEM image is not easy to read. The information of terrain elevation is contained in the image samples. The color image, however, shows the complexity of pictorial information.

From the perspective of image information mining both types of images, pictorial and multidimensional signals give rise to the same problem. Their understanding depends on the accuracy of:

- information content modelling,

- modelling the users understanding.

Thus, image information mining can be seen as a communication task. The source of information is the large heterogeneous image archive. The receiver is the community of users. The accuracy of communication, i.e. the success of finding the information needed as exploration results, depends on the accuracy of the previously assumed levels of modelling.

\section{Information mining: concept}

We developed a theoretical concept for image information representation and adaptation for the user conjecture (DATCu et al. 1998; Datcu et al. 1999; Datcu \& SEIDEL 1999; SCHRÖDER et al. 1998; SCHRÖDER et al. 2000). A quasi-complete description of the image content is obtained by utilization of a library of models. The feature extraction basically splits the image content into different information channels. An unsupervised clustering is done for each information channel as an information encoding and data reduction operation. Then, during the operation of the system, an interactive learning process allows the user to create links, i.e. to discover conditions between the low-level signal description and the target of the user.

The image features reflect the physical parameters of the imaged scene, thus, assuming the availability of certain models, the scene parameters can be extracted. For example, color and image texture carries information about the structure of object surfaces. However, in the case of modelling high complexity signals, a large 

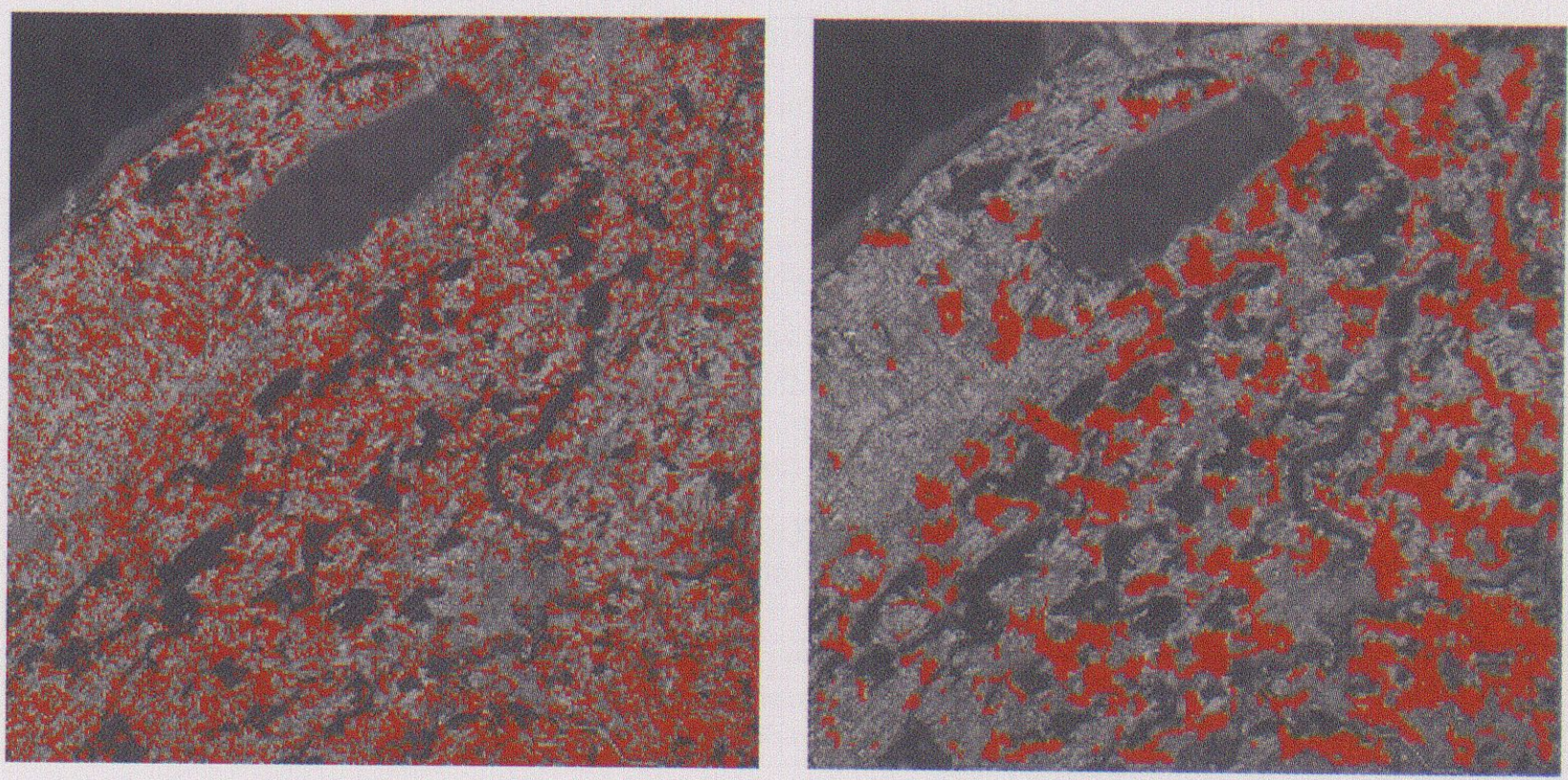

Fig. 3: Example of image information content extraction in a Landsat-TM image of Switzerland

Explanation: Left: Spectral image content (in red) obtained by the correlation of a specified cluster model with the pixel position in the image. Right: Texture image content obtained in similar manner. Here, however, the textural information characterizes structures, thus the resulting classification has connected areas. The information is indexed enabling the discovery of all images with similar spectral or textural properties.

Beispiel der Bestimmung des Bildinformationsgehalts in einer Landsat-TM Szene in der Schweiz Exemple d'extraction de contenu de l'information d'image dans une image de Landsat-TM de la Suisse Source: http://isis.dlr.de/mining

number of sources coexists within the same system, thus multiple candidate models are needed to describe the information sources in the image. Also, to reduce complexity, to capture the class structure, to discover causalities and to provide computational advantages, the models are likely to be analyzed hierarchically. The hierarchical information representation is further presented and depicted in Fig. 2:

- Image data: the information is contained in the samples of the raw data. It is the lowest level of information representation.

- Image features: the performance of information extraction depends critically on the descriptive or predictive accuracy of the probabilistic model employed. Accurate modelling typically requires high-dimensional and multi-scale modelling. For non-stationary sources, accuracy also depends on adaptation to local characteristics. For a quasi-complete characterization of the image content, information is extracted in the form of parameters characterizing color or spectral properties, texture as interactions among spatially distributed samples and the geometrical attributes of image objects.

- Meta features: estimation of the image features requires the assumption of some data models. The type of model used, its evidence and complexity, offers a form of meta information, e.g. it describes the quality of the extracted parameters. From a data aggregation perspective, a meta feature is an indicator of information commensurability, e.g. estimated texture features using co-occurence matrix are not comparable with parameters of Markov random fields. The meta features have semantic value.

- Cluster model: the signal features have n-dimensional representations. Due to observation noise or model approximations the feature space is not occupied homogeneously. Another level of information abstraction focuses on the type of feature grouping, e.g. the cluster models and their associated parameters. The obtained clusters only represent information for each category of the features.

- Semantic representation: it is known that the distinction between the perception of information as signals and symbols is generally not dependent on the form in which the information is presented but rather on the conjecture in which it is perceived, i.e. upon the hypothesis and expectations of the user. Augmentation of data with meaning requires a higher level of abstraction. The extracted information, represented in the form of classes, is fused 
A Bayesian Approach for Image Information Mining.

A Bayesian stochastic model is the tuple

$$
\{\mathrm{p}(\mathrm{y} \mid \mathrm{x}), p(x)\}
$$

where $p(y \mid x)$ is the parametric likelihood model and $p(x)$ is a prior distribution defined in the parameter space. The Bayesian Theorem

$$
p(x \mid y)=\frac{p(y \mid x) p(x)}{p(y)}
$$

shows how the information in $\mathrm{x}$ is updated by the information contained in the data $\mathrm{y}$.

A Bayesian hierarchical model assumes the prior $\mathrm{p}(\mathrm{x})$ is decomposed in conditional distributions

$$
\left\{p\left(x \mid \Theta_{1}\right), p\left(\Theta_{1} \mid \Theta_{2}\right), \ldots, p\left(\Theta_{\mu}\right)\right\}
$$

where $p\left(\Theta_{\mu}\right)$ is the marginal distribution.

From this perspective information mining in image archives is defined as a Bayesian learning of a hierarchic model

$$
\{p(I \mid \omega) p(\omega \mid L)\}
$$

which explains the dependencies between the image data $\{I\}$, the image content extracted with different models $\{\omega\}$ and the semantic labels $\{L\}$ defined by the user.

The inference is carried on as a Bayesian network, as presented in the figure below.

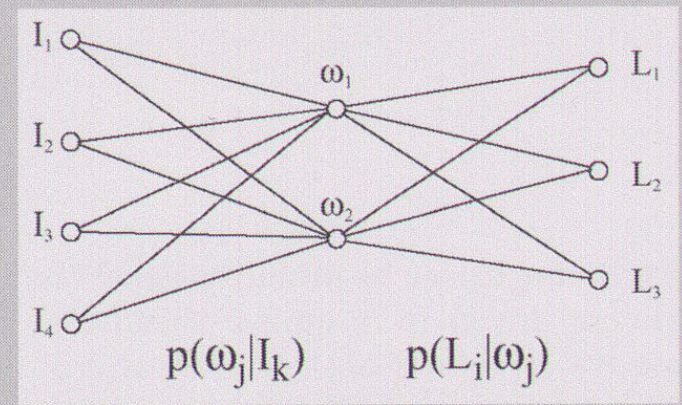

Three mining functions can be implemented:

1. the search of all images containing a target semantic label

$$
p(L \mid\{I\})
$$

2. the discovery of the stochastic image models explaining a given semantic level

$$
p(L \mid\{\omega\})
$$

3. the search of all images containing a certain model

$$
\mathrm{p}(\omega \mid\{I\})
$$

The three mining functions are exploiting the hierarchical causalities modeled by a Bayesian network.

Insert 2: A Bayesian approach for Image Information Mining Ein Bayesischer Ansatz für das Image Information Mining 

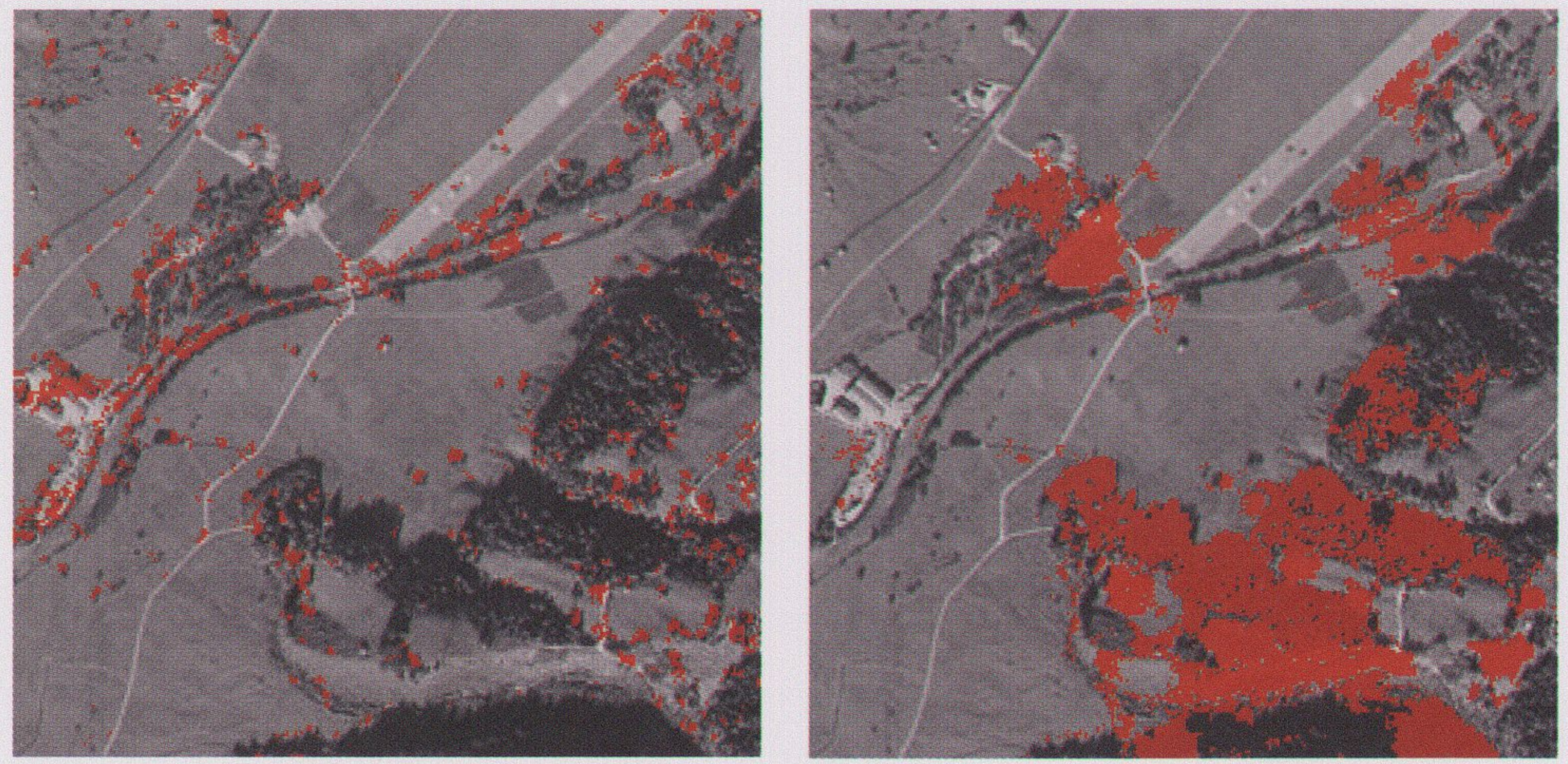

Fig. 4: Scale selection in aerial photography

Explanation: On the left, the structures in the image correspond to a fine scale. On the right, the same image may be seen, but at a rougher scale. The scale of structures in images is a fundamental descriptor, both in relation to the visual interpretation, and objectively in relation to the resolution of the sensor. The parameters of a multiscale random file are used to automatically detect the relevant scales. The information is indexed enabling the discovery of all images with structures at similar scales.

Skalenauswahl in Luftbildern

Sélection d'échelles en photographie aérienne

Source: http://isis.dlr.de/mining

in a supervised learning process. Prior information in the form of training data sets or expert knowledge is used to create semantic categories by associations to different information classes. Thus, the observations are labelled and the contextual meaning is defined.

In order to implement the hierarchical representation of the image information content, the data is pre-processed. First, the image features are extracted for different image scales. Then, the image features are clustered, before a signal content index is created using the cluster description, the scale information and the type of stochastic model assumed for the image parameters. A Bayesian learning algorithm allows a user to visualize and to encapsulate interactively his prior knowledge of certain image structures and to generate a supervised classification in the joint space of clusters, scales, and model types. The index of each image pixel is encoded by the spatial correspondence of the class information. The user is enabled to attach his meaning to similar structures occurring in different images, thus adding a label in the archive inventory. This label is further used to specify queries. The hierarchical information, meta-information, associations and semantic labels are stored and managed by a Data Base Management System (Insert 2).

\subsection{Mining driven by primitive signal features}

Mining driven by primitive signal features, such as spectral signatures or structural patterns, is enabled by the exploration of the links between the cluster and image data levels. Examples of spectral and textural signature mining are depicted in Fig. 3. Spectral mining is an example of physical, quantitative model exploration. For the Landsat-TM images used in the example only 6 spectral bands were selected.

\subsection{Theoretical criteria of mining information}

In the exploration of large image archives with rich information content it is important to group the data according to various objective information criteria. This helps the users to orient themselves within the search process.

One important characteristic is the scale at which relevant information is concentrated. We used a multiscale stochastic process for automatic scale detection and segmentation (REHRAUER et al. 1999; REHRAUER \& Dactu 2000). An example is shown in Fig. 4. The exploration of image archives by scale is a process which implicitly uses a priori knowledge assumed by the user, i.e. the ratio of the image resolution and size of objects he or she is searching for. 

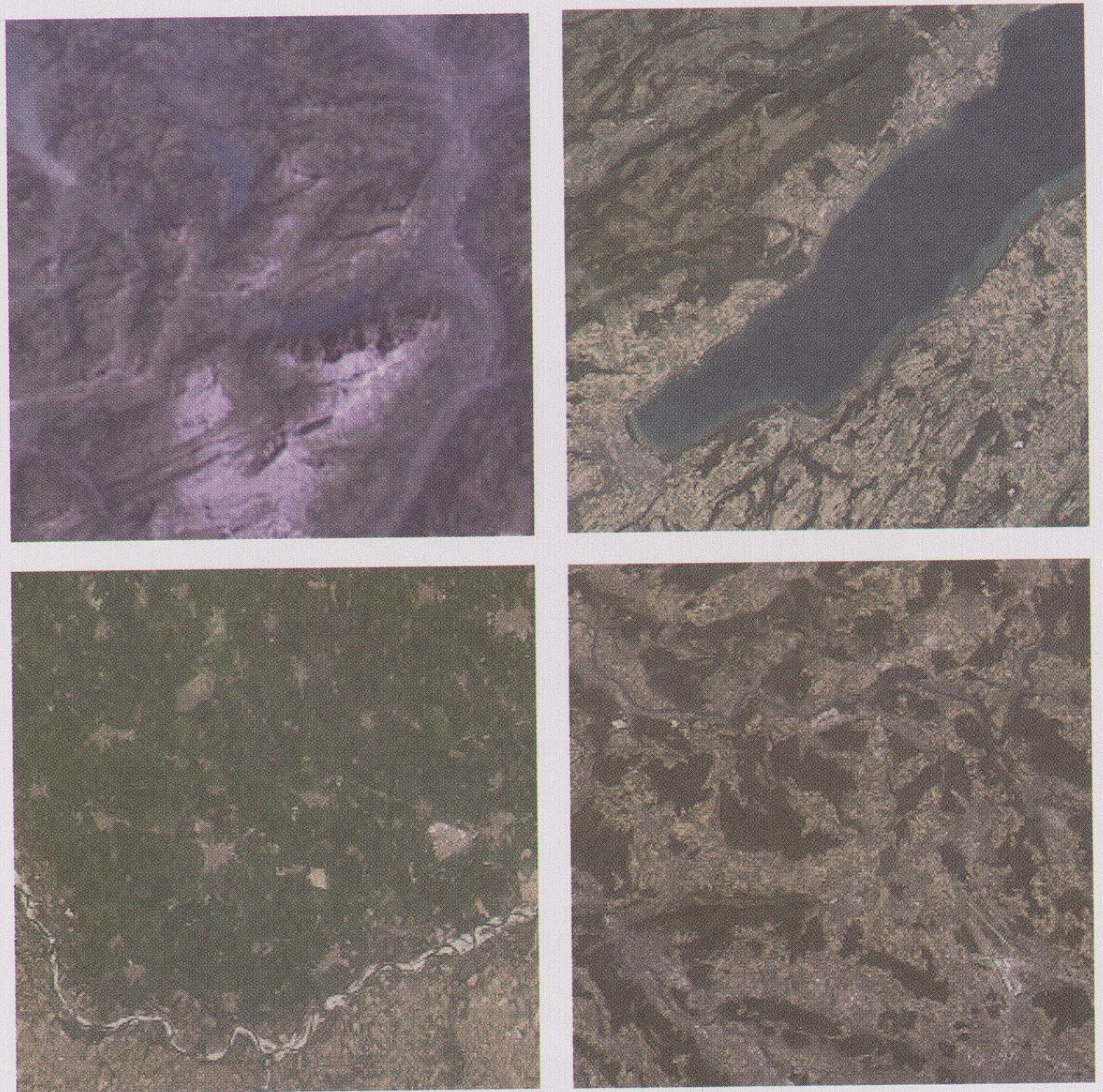

Fig. 5: Image complexity

Explanation: Top: Example of images of low (left) and high (right) spectral complexity. Bottom: Example of images of low (left) and high (right) structural complexity. The complexity of the images was measured as Kullback-Leiber entropy at the classification and clustering levels in the information hierarchy. The low complexity images are poor in information content, high complexity images show more «activity» thus giving a better chance to discover «interesting» structures or objects. The complexity values are indexed, enabling the discovery of all images with a similar behavior.

Bild-Komplexität

Complexité d'image

Source: http://isis.dlr.de/mining

The complexity of images is another information theoretical criteria used to rank images. The complexity is defined as the Kullback-Leiber divergence between the cluster level and the image data level. The complexity depends on the quality and type of model used. In Fig. 5, images are compared according to their spectral and textural complexity.

\subsection{Mining by interactive learning}

Interactive learning is the process of discovering the links between the user interest (target), the image content in terms of describing models and the images containing the assumed structure (DACTU et al. 1999; SCHRÖDER et al. 2000). In a first step, interactive learning uses a Bayesian network to create the links between the concept and cluster levels. During interactive learn- 


\section{Interactive Learning and Search}

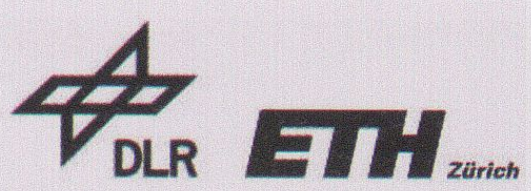

\section{Online training of new_label using two features (gmrf14.:spectr6.)}

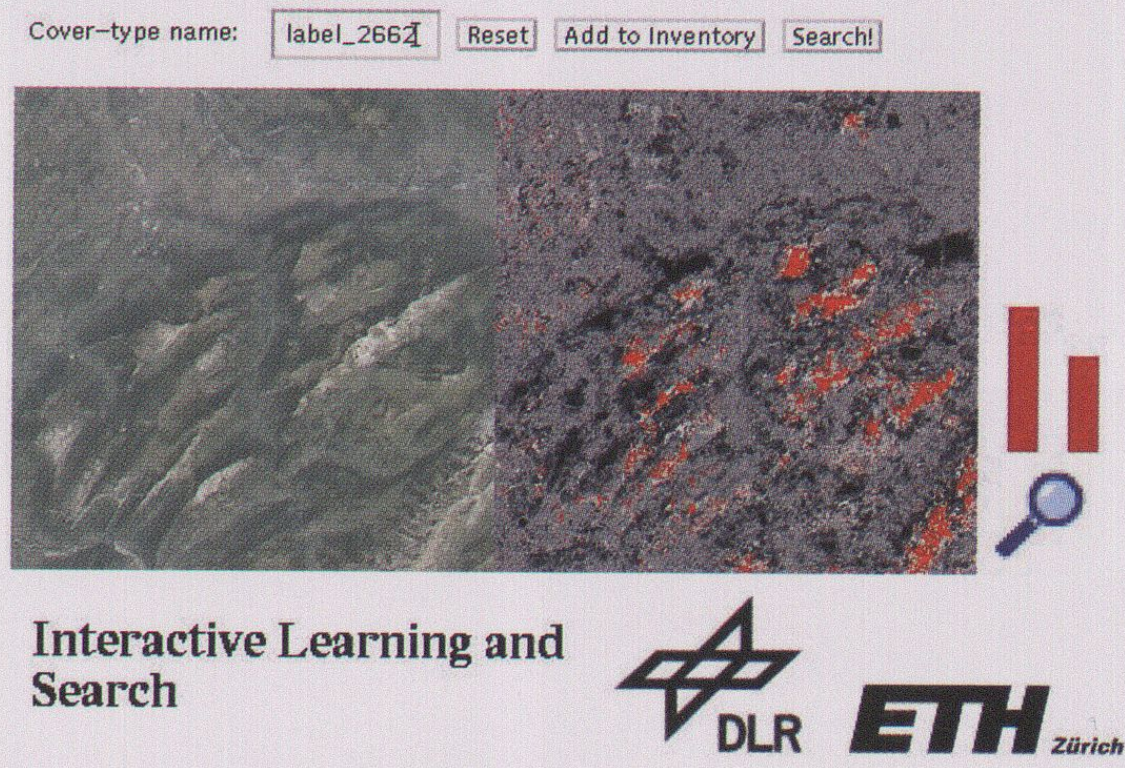

\section{Online training of alps using two features (gmrf11.WS03:gmrf11.WS04)}

$\begin{array}{llll}\text { Cover-type name: } & \text { Ralps Reset Add to Inventory Search! }\end{array}$

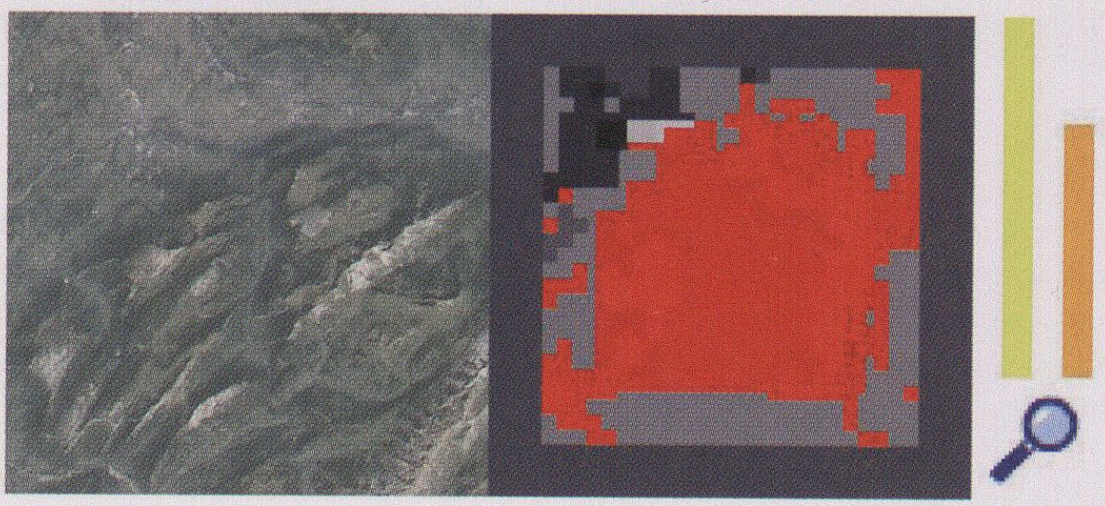

Fig. 6: Interactive training

Explanation: Top: Interactive training using fusion of spectral and textural information at the finest image scale. The target semantics is «meadow». Bottom: Interactive training using fusion of textural information estimated for the scales 1:2 and 1:3. This is the same image as before, but the target semantics is «mountain». Interactive learning is a form of information mining which enables adaption to the user conjecture. It is a purely exploratory function based on learning, fusion and classification processes, using the pre-extracted primitive attributs of an image, and allowing an open, very large semantic space. The user defined target is generalized over the entire image archive, thus allowing further exploration.

Interaktives Training

Apprentissage interactif

Source: http://isis.dlr.de/mining 


\section{Probabilistic Search Result for label_2662}

\section{A. Images with highest coverage of label_2662 (Click on an image to continue learning on it):}
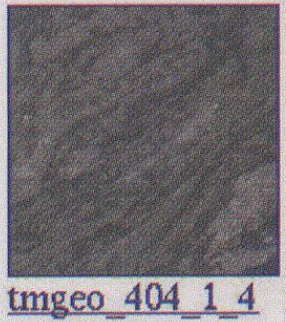

$24 \%$

label_2662

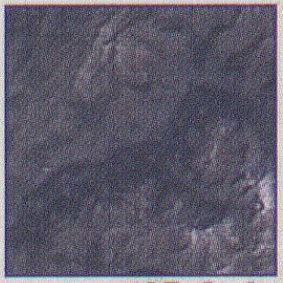

tmgeo 42726

$14 \%$

label 2662

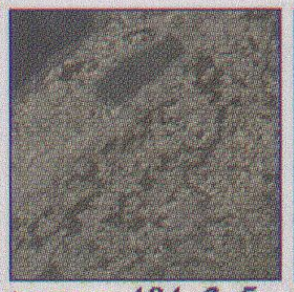

tmgeo 40425 $18 \%$ label 2662

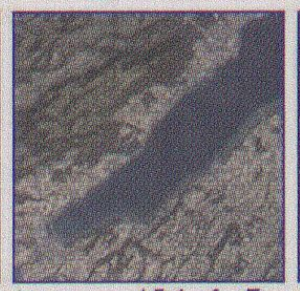

tmgeo 40415 $13 \%$ label 2662

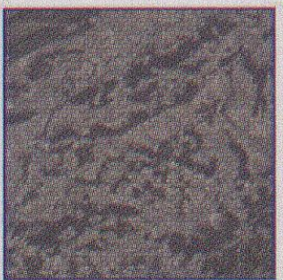

tmgeo 40434 $16 \%$

label 2662

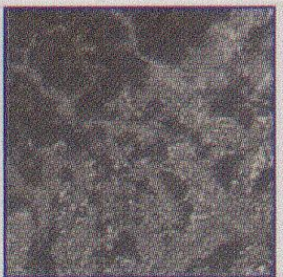

tmgeo 40421 $13 \%$

label 2662

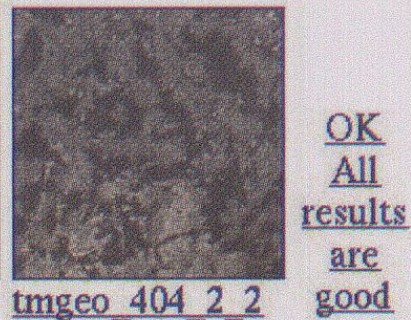

$15 \%$

label_2662

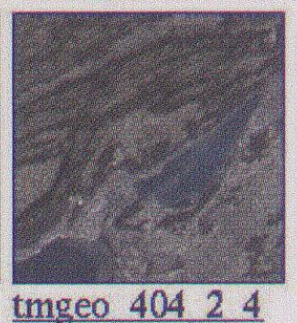

$13 \%$

label 2662

\section{Probabilistic Search Result for alps}

\section{A. Images with highest coverage of alps (Click on an} image to continue learning on it):
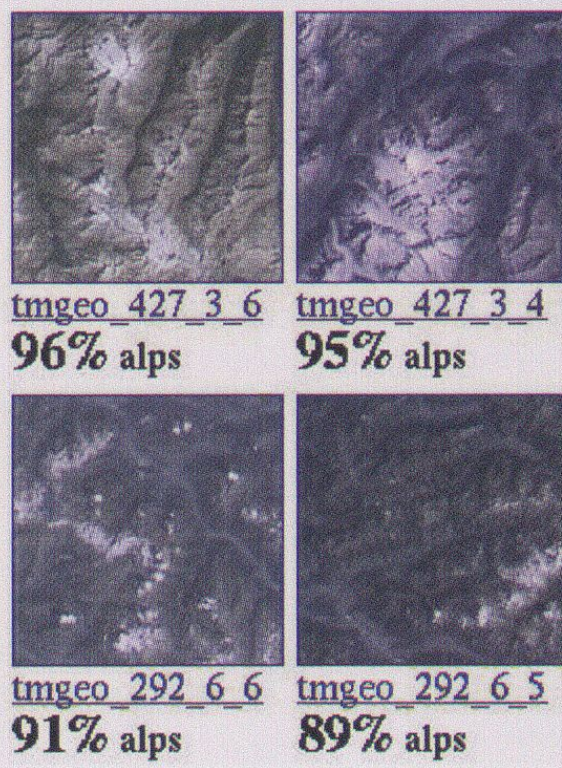

$95 \%$ alps

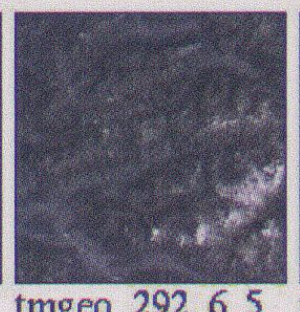

$89 \%$ alps
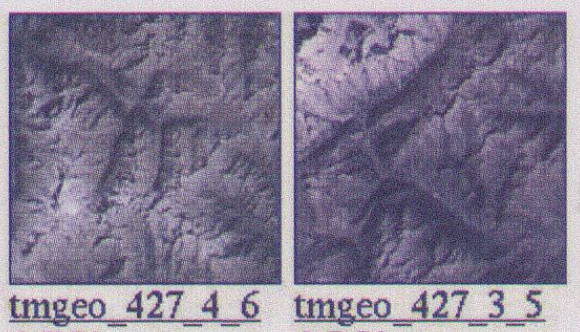

OK

All

results

are

good
$93 \%$ alps

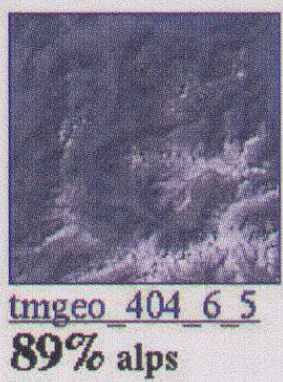

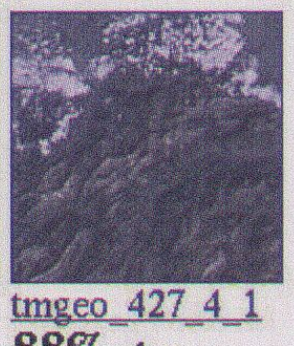

$88 \%$ alps

Fig. 7: Probabilistic search

Explanation: Top: The result of a probabilistic search for images containing «meadow». Bottom: The result of a probabilistic search for images containing «mountains». Both query results correspond to the interactive training as defined in Fig. 6.

Wahrscheinlichkeitssuche

Recherche probabiliste

Source: http://isis.dlr.de/mining 

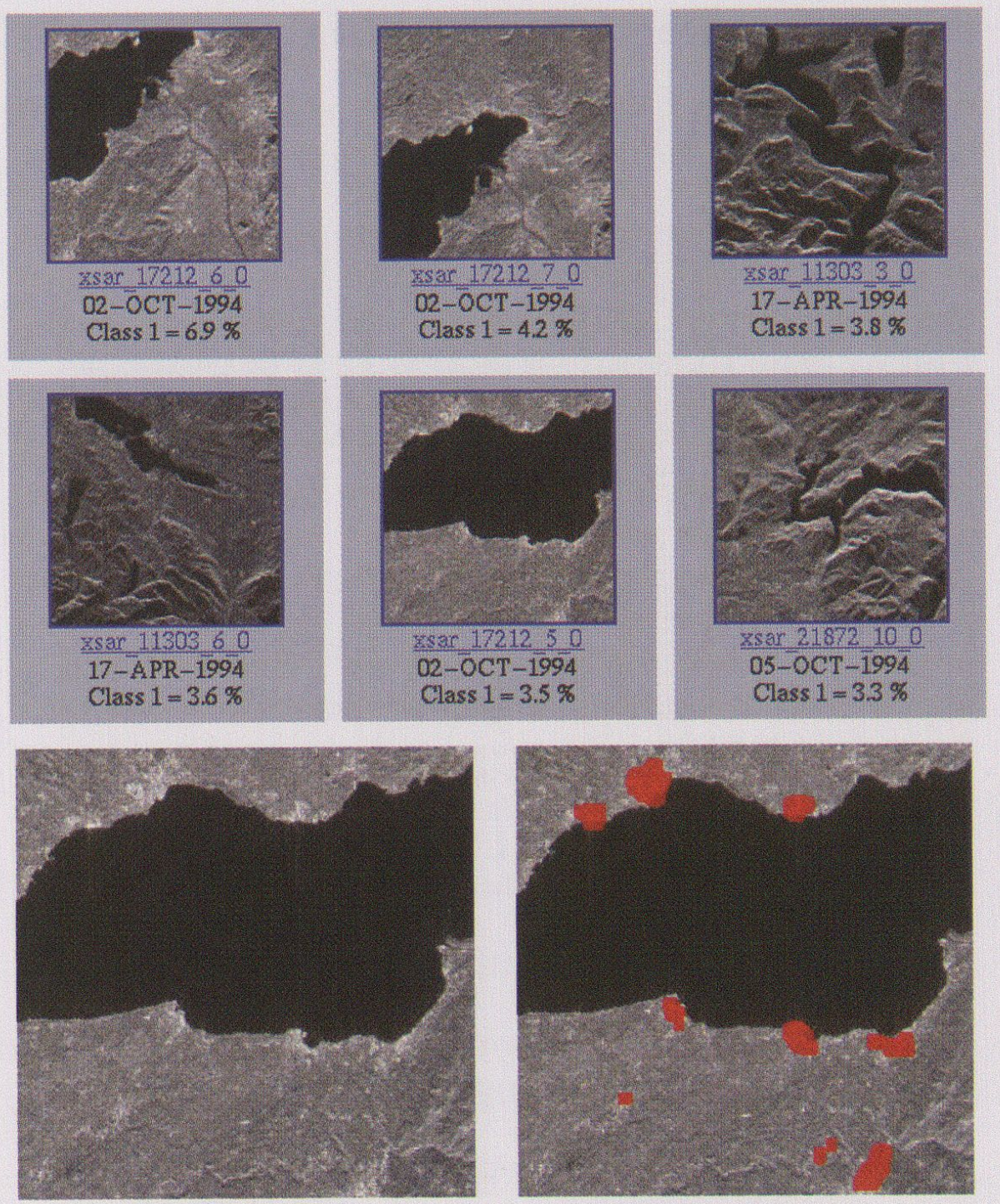

Fig. 8: Semantic CBIR on Synthetic Aperture Radar X-SAR SRL images of Switzerland

Explanation: Top: Result of semantic query - discovering settlements. The images have been automatically analyzed at ingestion in the archive, and a catalog entry was created for all images containing built up areas. Bottom: Each image has attached to it the result of the classification. The regions marked in red correspond to villages and cities, thus the result of the search is a list of images, augmented by the expected semantic image content.

Semantische inhaltsorientierte Bildabfrage anhand von Synthetic Aperture Radar-Daten (X-SAR SRL) der Schweiz CBIR sémantique sur des images de radars à ouverture synthétique X-SAR SRL de la Suisse

Source: http://isis.dlr.de/mining

ing the image data (quicklooks) are used to give examples and to index the spatial position of the target structures. In a second step, also using a Bayesian approach, a probabilistic search over the image space is performed. At this stage the links between the concept level, clusters and image data levels are created. The learning process uses positive and negative examples, both from the user and computer. It is a man-machine dialog.

In Fig. 6, an example is presented for the exploration of different models (texture at various scales and spec- tral signatures) to discover different semantic objects in the data.

The results of the probabilistic search are depicted in Fig. 7 for the cases indicated in Fig. 6.

\subsection{Semantic Content Based Image Retrieval}

Following automatic processing at data ingestion or in a semi-automatic manner using an interactive learning process, the system can create links between the concept level and the image data and cluster levels. 


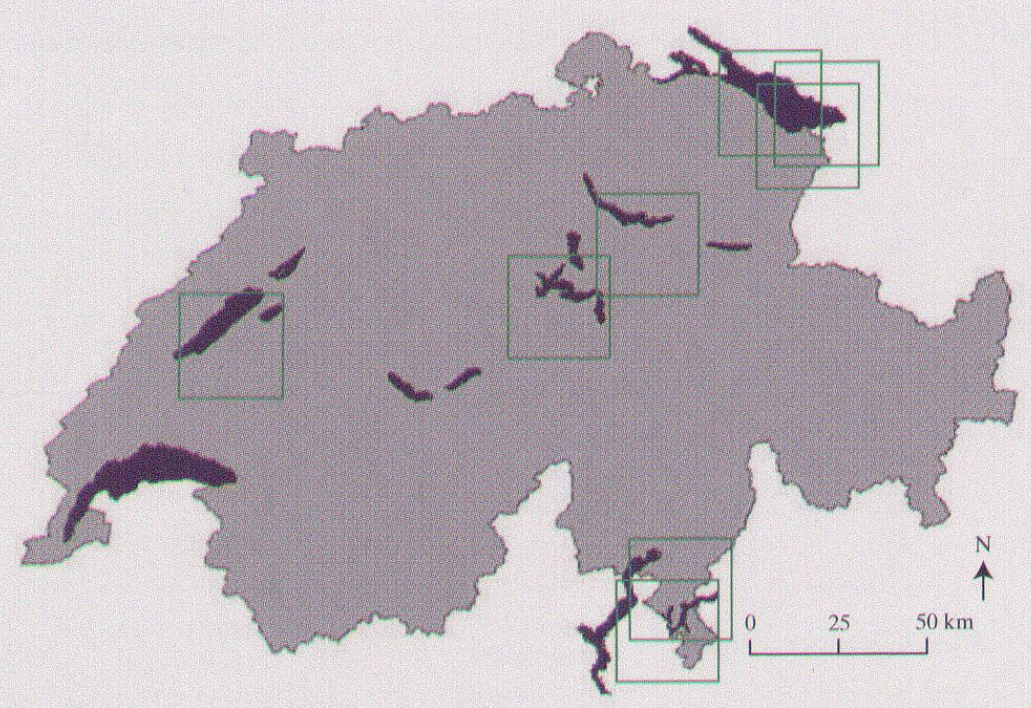

Fig. 9: The geographical location of the images obtained as a result of the semantic query (Fig. 8) Geographische Lage der Bilder gemäss der semantischen Abfrage (Fig. 8)

La localisation géographique des images obtenues comme résultat de l'interrogation sémantique (fig. 8)

Source: http://isis.dlr.de/mining

The user is enabled to specify semantic queries at the concept level and the system returns all images with the specified content, including a classification on individual images. An example is given in Fig. 8.

In the case of Earth Observation the geographical location is also used as meta-information allowing the location of the intensity images as indicated in Fig. 9, to be found.

\subsection{Knowledge driven image information mining and user conjecture}

During the interactive learning and probabilistic search the database management system (DBMS) holds a record of:

- the user semantic,

- the combination of models able to explain the user's target,

- the classification of the target structure in each individual image,

- a set of statistical and information theoretical criteria reflecting the effectiveness of the learning process.

The information and associations collected represent a pool of knowledge harvested in this manner or learned from the various system users. This information is used for other mining tasks and is itself object of mining, e.g. grouping of semantic levels, relevance feedback, joint grouping between the semantic space and the statistical or the information theo- retical measurement of the standard of the learning process.

\section{System architecture of information mining}

The system is implemented in a server-client architecture as presented in Fig. 10.

This concept was implemented and successfully demonstrated with an on-line experimental system (see http://isis.dlr.de/mining). The novel mining functions currently provided by the system may also be seen in the diagram.

\section{Conclusions}

We were able to develop a new concept for image information mining which puts a user centered perspective in the foreground, thus highlighting the communicative aspect of information ming. The hierarchy of information representation in conjunction with the quasi-complete image content description, enables implementation of a large variety of mining functions. The concept was demonstrated for a variety of Earth Observation data. Further work is being performed for the development of intelligent satellite ground segment systems and value adding tools. However, its potential is broad and other fields of applications are possible, such as medical imagery, biometrics, etc. 


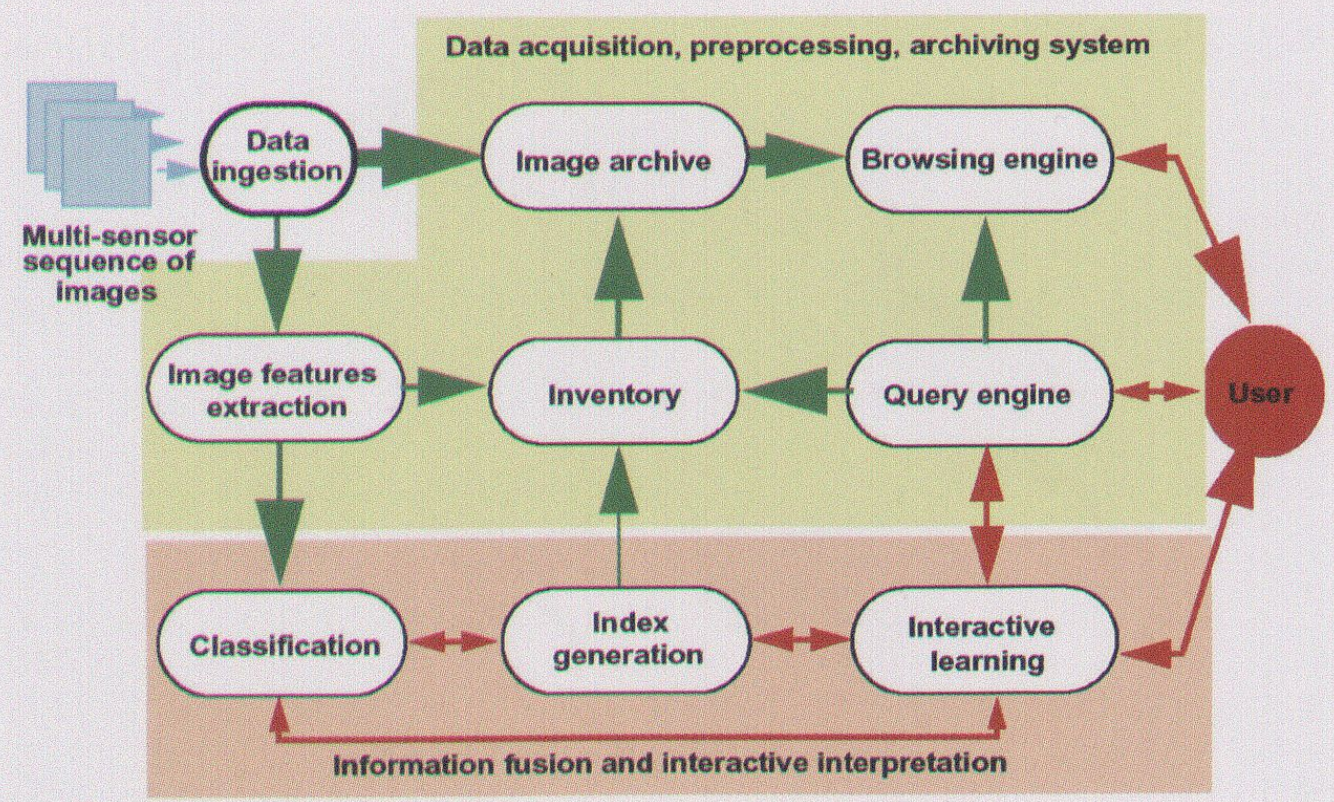

Fig. 10: The system architecture. In yellow the server, violet the client.

System-Architektur. Gelb der Server, violett der Nutzer.

L'architecture de système. En jaune le serveur, en violet le client.

Graphics: Image Information Mining (Cooperation DLR/ETHZ)

The proposed concept is far away from being fully exploited. Presently ongoing theoretical development is profoundating the problematic of image complexity. In the case of high heterogeneity observations the complexity and the course of dimensionality are two key issues which can hinder the interpretation. There- fore, as an alternative solution to the «interpretation», we propose an exploratory methodology approached from a information theoretical perspective in a Bayesian frame.

Another direction is the analysis of cluster models from

In recent years most of the available satellite images over the territory of Switzerland have been stored in the Swiss National Remote Sensing Image Archive. This archive was initiated and is maintained by the National Point of Contact (NPOC) of Switzerland in cooperation with the Swiss Federal Office of Topography and the Remote Sensing Labs (RSL) at the University of Zürich (www.npoc.ch).

The archive is installed at the Centro Svizzero di Calculo Scientifico (CSCS, Swiss High Performance Computer Center) in Manno which belongs to the Swiss Federal Institute of Technology (ETH).

As a result of an ongoing research activity at the Computer Vision Lab (ETH), a MultiMission Demonstrator (MMDEMO) has been set up in order to show the advantages as soon as more sophisticated catalogue systems become available. A variety of datasets of the national archive are used in the demonstrator for content-based querying and retrieval techniques: http://www.vision.ee.ethz.ch/ rsia/ 
the perspective of an «objective» semantic approach, aiming at the elaboration of methods to understand the nature of the feature space.

A direction of application of the developed methodology is the mining of temporal series of images, considering the integration of spatio-temporal signal analysis.

Even the concept of learning the user conjecture was to some extent demonstrated. Difficult areas, such as developing image grammars and representation of image content in different contextual environments are under further research. The former is a semantic problem which can arise between different users when they define or describe the same structures differently, requiring the primitive attributes, features, domains, values or causalities to be translated.

A number of challenges, mainly in the design of multidimensional DBMS, man-machine interfaces and distributed information systems, will probably be investigated soon (Insert 3).

\section{Acknowledgement}

The project was supported by the Swiss Federal Institute of Technology (ETH), Research Foundation Advanced Query and Retrieval Techniques for Remote Sensing Image Archives (Grant: RSIA 0-20255-96). The authors would like to thank Michael Schröder and Hubert Rehrauer for converting the concept into algorithms and setting up the Multi-Mission Demonstrator (MMDEMO).

\section{References}

Cox, I.J., Miller, M.L., OMohundro, S.M. \& P.N. YiANILos (1996): PicHunter: Bayesian Relevance Feedback for Image Retrieval. - Proceedings of the International Conference on Pattern Recognition, Vienna, Austria. Datcu, M.,Seidel, K. \& M. Walessa (1998): Spatial Information Retrieval From Remote Sensing Images: Part I. Information Theoretical Perspective. - IEEE Transactions on Geoscience and Remote Sensing 36: 1431-1445. Datcu, M., Seidel, K. \& G. Schwarz (1999): Elaboration of advanced tools for information retrieval and the design of a new generation of remote sensing ground segment systems. - In: KANELLOPOULOS, I. (ed.): Machine Vision in Remote Sensing. - Berlin, Hamburg: Springer: 199-212.

Datcu, M. \& K. Seidel (1999): Bayesian methods: applications in information aggregation and data mining. - International Archives of Photogrammetry and Remote Sensing 32, Part 7-4-3 W6: 68-73.

Datcu, M., Seidel, K., D'Elia, S. \& P.G. Marchetti (2002): Knowledge-driven Information-Mining in remote sensing image archives. - ESA (European Space Agency) Bulletin.
Minka, T.P. \& R.W. Picard (1997): Interactive learning with a society of models. - Pattern Recognition 30: 565-581.

Rehrauer, H., Seidel, K. \& M. Datcu (1999): Multiscale indices for content-based image retrieval. - In: Proceedings of 1999 IEEE International Geoscience and Remote Sensing Symposium IGARSS'99, volume V: 2377-2379.

Rehrauer, H. \& M. Datcu (2000): Selecting scales for texture models. - In: PIETIKÄINEN, M.K. (ed.): Texture analysis in machine vision. - Series in machine perception and artificial intelligence 40, World Scientific.

Schröder, M., Rehrauer, H., Seidel, K. \& M. Datcu (1998): Spatial Information Retrieval From Remote Sensing Images: Part II. Gibbs Markov Random Fields. - IEEE Transactions on Geoscience and Remote Sensing 36: 1446-1455.

Schröder, M., Rehrauer, H., Seidel, K. \& M. Datcu (2000): Interactive learning and probabilistic retrieval in remote sensing image archives. - IEEE Transactions on Geoscience and Remote Sensing 38: 2288-2298 VeltKamp, C.R., Burkhardt, H. \& H.-P. Kriegel (eds) (2001): State-of-the-Art in Content-Based Image and Video Retrieval. - Köln: Kluwer.

Zhang, J., Hsu, W. \& M.L. LeE (2001): Image Mining: Issues, Frameworks and Techniques. - In: Proceedings of the Second International Workshop on Multimedia Data Mining (MDM/KDD’2001), San Francisco, CA, USA, August, 2001.

\section{Summary: Image Information Mining - Exploration of Earth Observation Archives}

The new generation of high resolution imaging satellites acquires huge amounts of data which are stored in large archives. The state-of-the-art systems for data access allow only queries by geographical location, time of acquisition or type of sensor. This information is often less important than the content of the scene, i.e. structures, objects or scattering properties. Meanwhile, many new applications of remote sensing data are closer to computer vision and require the knowledge of complicated spatial and structural relationships among image objects.

We are creating an intelligent satellite information mining system, a next generation architecture to help users to rapidly collect information, a tool to enhance and to manage the huge amount of historical and newly acquired satellite data-sets by giving experts access to relevant information in an understandable and directly usable form and to provide friendly interfaces for information query and browsing.

Research topics are within the frame of Bayesian learning, content-based querying, data modelling and adaptation to user conjecture. 


\section{Zusammenfassung: Image Information Mining - Erforschung von Erderkundungsarchiven}

Die neue Generation hochauflösender Satellitenbilder liefert grosse Datenmengen, die in entsprechend grossen Archiven gespeichert werden. Moderne Katalogsysteme erlauben nur Abfragen nach dem geographischen Ort, der Aufnahmezeit oder dem Sensortyp. Diese Information ist nun oftmals weniger wichtig als der Inhalt einer Szene, wie er durch Strukturen, Objekte oder deren Streuverhalten gegeben ist. In der Zwischenzeit gehören viele neue Fernerkundungsanwendungen eher zu computer vision, denn sie benötigen das Wissen über die recht komplizierten räumlichen und strukturellen Zusammenhänge zwischen den Objekten.

Wir bauen ein intelligentes Informationsabfragesystem für Satellitenbilder auf. Mit einer neuen AbfrageArchitektur wollen wir den Datennutzer einmal bei der Auswahl der Fernerkundungsbilder unterstützen, die für seine Aufgaben geeignet erscheinen, zum anderen sollen Werkzeuge bereitgestellt werden, welche die bessere Nutzung der Daten gewährleisten und die es erlauben, der ausserordentlich grossen Datenmenge Herr zu werden, die durch die historischen und neuen Datensätze zur Verfügung stehen. Dazu soll den Experten die relevante Bildinformation mit Hilfe von benutzerfreundlichen Abfragemenus in einer verständlichen und direkt nutzbaren Form angeboten werden. Die Forschung liegt in den Bereichen des Bayesian Lernens, inhaltsorientierter Abfragetechniken, Datenmodellen und der Berücksichtigung des Benutzerverhaltens.

\footnotetext{
Résumé: Extraction d'information provenant d'images - Exploration des archives d'observation de la terre

La nouvelle génération d'images satellite haute résolution permet d'acquérir une importante quantité de données qui est stockée dans d'importantes archives. Les systèmes dernier cri pour l'accès aux données permettent seulement des questions par emplacement géographique, période d'acquisition ou type de sonde. Cette information est souvent moins importante que la teneur de la scène, c'est-à-dire structures, objets ou leurs propriétés de dispersion. En attendant, beaucoup de nouvelles applications des données de télédétection sont plus près de la vision par ordinateur et exigent la connaissance des rapport spatiaux et structuraux complexes parmi des objets d'image.
}

Nous créons un système intelligent d'extraction de l'information satellite, une architecture de prochaine génération pour aider les utilisateurs à recueillir rapidement l'information dans le cadre de leur projet. Nous créons aussi un outil destiné à faciliter la maîtrise de l'extraordinaire quantité de donnnées désormais disponibles, tant à valeur historique que présente. Nous comptons faciliter aux experts l'accès à l'information appropriée sous une forme compréhensible et directement utilisable en vue de la fourniture des interfaces accessibles à l'information et la lecture rapide. Les sujets de recherche sont structurés autour de la théorie Bayesienne de l'interrogation basée sur le contenu, de la modélisation de données et de la prise en compte du comportement des utilisateurs.

\section{Teaching of Geography - pertinent questions}

- How is Image Information Mining defined in comparison with the fields of Data Mining, Image Analysis, and Content Based Image Retrieval?

- Which are the hierarchical levels of image information representation, and which is the role of the hierarchy?

- Imagine and design scenarios for utilization of the Image Information Mining concepts in your field of application.

Prof. Dr. Mihai Datcu, German Aerospace Center DLR, Remote Sensing Technology Institute - IMF, Oberpfaffenhofen, D-82234 Wessling.

e-mail:mihai.datcu@dlr.de

Dr. Klaus Seidel, Remote Sensing Group, Computer Vision Lab ETHZ, Gloriastrasse 35, CH-8092 Zürich. e-mail: seidel@vision.ee.ethz.ch
Manuskripteingang/received/manuscrit entré le 17.2.2003
Annahme zum Druck/accepted for publication/accepté pour l'impression: 5.6.2003 